\title{
Wireless Communication and Its Effect to Tunneling Giant Magneto- Resistive (TGMR) Reader in Head Gimbal Assembly Process
}

\author{
Dutharuthai Na Pombejara $^{1,3}$, Chaiyan Jettanasen ${ }^{2}$, Kraisorn Rittipad ${ }^{3}$ \\ ${ }^{1}$ College of Data Storage Innovation, King Mongkut's Institute of Technology Ladkrabang, Thailand \\ ${ }^{2}$ Faculty of Engineering, King Mongkut's Institute of Technology Ladkrabang, Thailand \\ ${ }^{3}$ Seagate Technology (Thailand) Ltd. Nakornratchasima, Thailand
}

\begin{abstract}
TGMR reader is current technology of magnetic recording read sensor which dramatically improves readout amplitude, speed and recording areal density. In advanced manufacturing environment, automated tools are increasingly used and wireless communication device is aimed to be employed to enhance the operation efficiency. However, electromagnetic fields from wireless communication devices could harm or damage the most energy sensitive devices/equipment, such as TGMR reader sensor, in head gimbal assembly process. This study surveys, therefore, electromagnetic interference from wireless communication devices using CTM048, EM Field Meter with CTS001 directional antenna and electromagnetic field module, then use Agilent 34410A to measure TGMR resistance and thermal change of TGMR head while placing in the field of wireless communication device. Build-in Differential-Ended Temperature Coefficient of Resistivity sensor is utilized for proximal TGMR temperature. Impact to electrical performance of TGMR pre and post actions simulating HGA assembly process will be confirmed using Quasi-static Tester.
\end{abstract}

\section{Introduction}

Nowadays wireless communication has been integrated to every activity owing to its convenience. Such interference may cause undesirable effects to sensitive equipment or sensitive devices. There were some studies about Electromagnetic Interference (EMI) effect to magnetic recording head, but none of them studied on real TGMR head. Previous studies about EMI effect from wireless communication were done on $5 \mathrm{~Gb} / \mathrm{in}^{2} \mathrm{PtMn}$ GMR sensors [1] [2] [3], while current technology is up over $500 \mathrm{~Gb} / \mathrm{in}^{2}$ sensor. A study mentioned about TGMR head, but no technical discussion or end result of TGMR sensor impact presented in the paper [4]. Newer studies had simulated TGMR sensor impact in stand-alone stage. The consideration of connection to slider body (Key components of Slider are Reader, Writer and Advance Air Bearing Surface), Trace-Gimbal Assembly (TGA), suspension or other assembly was excluded from simulation scope [5] [6]. A study concludes that magnetic heads in production can be exposed to electrical overstress exposure of different types due to the ultrasensitive nature of magnetic heads [7]. This study is to confirm if the electromagnetic fields from wireless communication devices will harm the most energy sensitive devices, such as TGMR recording reader sensor, in assembly process.

\section{Structures and Process}

\subsection{The difference between Giant Magneto- Resistive (GMR) and Tunneling Giant Magneto- Resistive (TGMR) head structure}

GMR Current-in-Plane (CIP) architecture has been replaced by TGMR Current-Perpendicular (CPP) architecture to allow higher data recording density

Key distinguishing element of TGMR is Magnetic Tunnel Junction (MTJ), which consists of two ferromagnets separated by an angstrom-thin insulator. Electrons can tunnel from one ferromagnet to the other if the barrier is thin enough. With this quantum mechanical phenomenon, the MTJ can be switched between two states of electrical resistance, high resistance and low resistance, then provides higher MR Ratio $\left(\Delta R / R_{0}\right.$ : change of TGMR resistance/minimum resistance of TGMR), higher readout amplitude and speed as well as lower areal resistance ( $R A$ : Resistance $\mathrm{x}$ Area) and head noise [8].

TGMR resistance is ten times higher than GMR resistance. This makes TGMR undergoing ten times higher joule heat than GMR. The angstrom-thin metal oxide barrier makes TGMR more susceptive to external energy. Fortunately, not like GMR, the TGMR head do not have insulator between buffer and shield, so TGMR can dissipate thermal directly through top and bottom 
metal shield. Example of simplified structure of GMR and TGMR recording reader head are explained in Figure1 and Figure2. Actual structure and material may be difference base on design and fabrication.

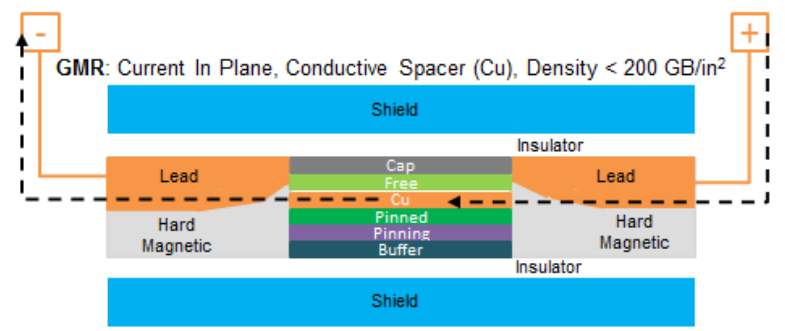

Figure1. Example of simplified GMR head structure.

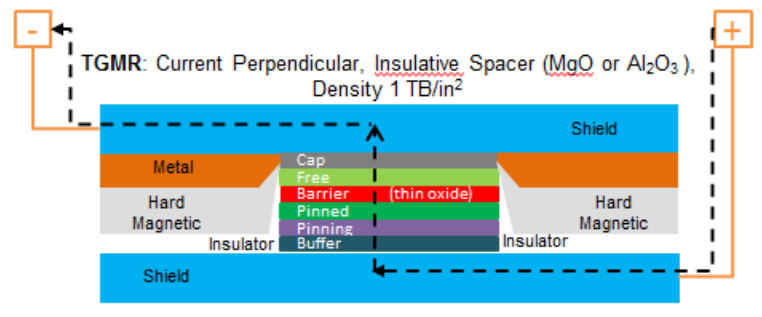

Figure2. Example of simplified TGMR head structure.

\subsection{Head-Gimbal Assembly and its process}

Head-Gimbal Assembly (HGA) is an important piece part of Hard Disk Drive in which slider body, which containing read-write head, located and flies above magnetic recording media.

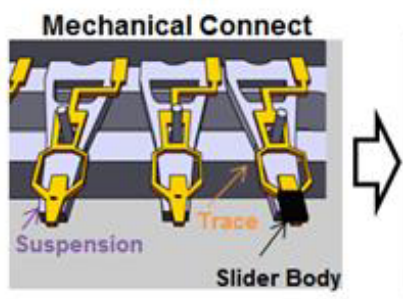

Electrical Connect
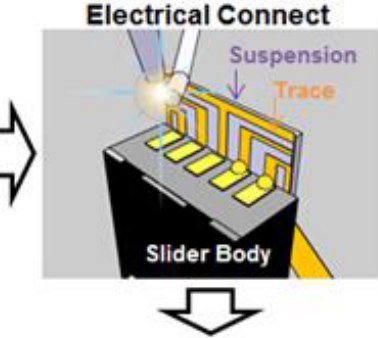

Electrical Test

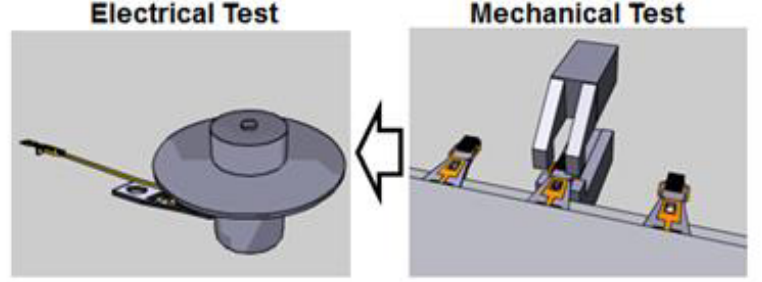

Figure3. Head-Gimbal Assembly process steps

The manufacturing process of Head-Gimbal Assembly is basically in full automation. As shown in Figure.3, robot end-effector pick-and-place each piece part, make mechanical connection and electrical connection between Trace-Gimbal Assembly (TGA) and Slider body. Then check performance via mechanical test and electrical test processes. Piece parts of HGA are contacted and in-contacted with grounded metal or static dissipative material many times along the automated process.

\section{Experiment and discussion}

\subsection{Wireless communication devices in factory environment - Electromagnetic Interference}

Electric Field from wireless communication devices is measured using CTM048, EM Field Meter with CTS001 directional antenna and electromagnetic field module. This measuring instrument has Peak and Average Measurement Range of $0.001 \mathrm{~V} / \mathrm{m}$ - $20.00 \mathrm{~V} / \mathrm{m}$, Frequency Bandwidth of $1 \mathrm{MHz}$ to $2.5 \mathrm{GHz}$, Antenna Factor Range of -40.0 to $40.0 \mathrm{dBm}-1$, Antenna Frequency Response of $10 \mathrm{MHz}-2.5 \mathrm{GHz}$. The measurement is performed in empty large shield room, at distance of 1 inches $(25.4 \mathrm{~mm})$ between CTS001 antenna and wireless communication devices, which are (1) Portable Two-way Radio Transceiver (walkie-talkie), (2) Wireless LAN Access Point (WiFi router), (3) GSM Cellular phone, (4) Cordless phone, (5) Laptop computer, and (6) Wireless mouse \& Keyboard set.

In Figure4, the result shows that E-Field of higher than $5 \mathrm{~V} / \mathrm{m}$ is observed from three types of wireless communication devices, Portable Two-way Radio Transceiver, GSM Cellular Phone and Cordless Phone. Whereas the E-Field of higher than $1 \mathrm{~V} / \mathrm{m}$ is observed

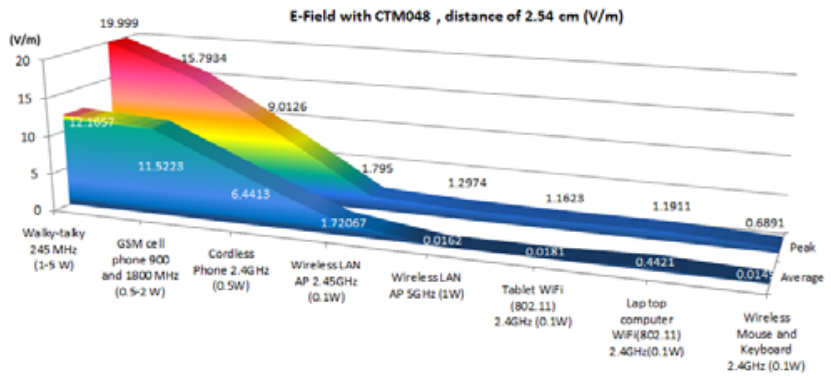

Figure4. Result of Electric field survey of wireless communication devices

\subsection{Thermal change and DC voltage of TGMR head while placing in the field of wireless communication device}

Build-in Differential-Ended Temperature Coefficient of Resistivity (DETCR) sensor [9] [10] has been used to observe actual thermal change at TGMR head. In this experiment, the build-in DETCR sensor was located inside the slider body, approximately 1 micron $\left(1 \times 10^{-6} \mathrm{~m}\right)$ from TGMR reader.

Agilent 34410A with shielded-cables and Gryphic probe was modified to measure DETCR resistance and DC voltage of TGMR while placing TGMR head in the field of wireless communication devices.

The response to local changes in temperature at the DETCR sensor is based on equation (1) and (2). The change of DETCR resistance $\left(\triangle R_{\text {DETCR }}=R_{\text {DETCR }}\right.$ $\left.R_{0 \_D E T C R}\right)$ is proportional to the change of DETCR 
temperature $\left(\triangle T_{D E T C R}=T_{D E T C R}-T_{0 D E T C R}\right)$ while $R_{0 D E T C R}$ is original resistance of the DETCR, $R_{D E T C R}$ is resistance of the DETCR at the point of time to compare, $T_{0}$ DETCR is original temperature of the DETCR, $T_{D E T C R}$ is temperature of the DETCR at the point of time to compare, and $\alpha$ is specific to DETCR material.

$$
\begin{gathered}
\Delta R_{D E T C R}=a \Delta T_{D E T C R} \\
R_{D E T C R}-R_{0_{-} D E T C R}=a *\left(T_{D E T C R}-T_{0 \_D E T C R}\right)
\end{gathered}
$$

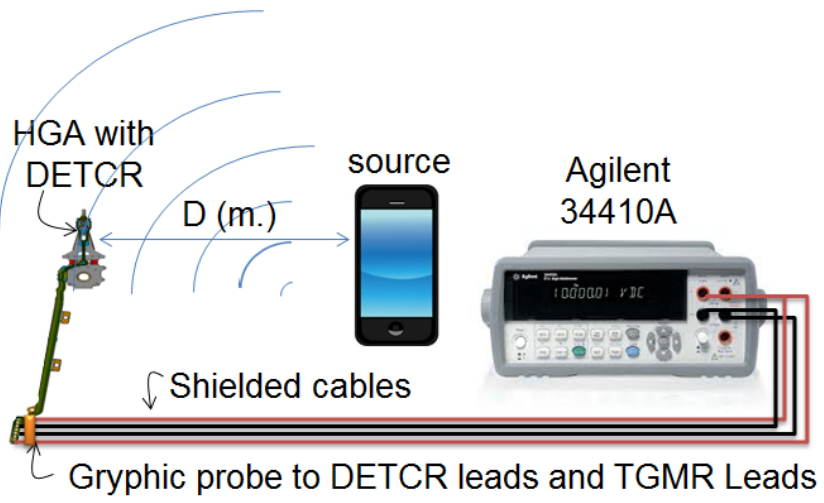

Figure5. Measurement set-up on Thermal change and DC voltage of TGMR head while placing in the field issued from wireless communication device

Result is shown in Figure6.1; the temperature changes when placing TGMR in the field of wireless communication device. The original temperature $\left(\mathrm{T}_{0}\right)$ is 22 degree Celsius and the change of temperature $(\Delta \mathrm{T})$ is less than 3 degree Celsius, so maximum temperature is less than 25 degree Celsius which is needed to be compared whether it is high enough to reach the threshold of soft level of stability failure of TGMR. Typically, the threshold should be more than 100 degree Celsius.

Figure6.2 shows measurement result of DC voltage drops on TGMR, while placing TGMR head in the field of wireless communication device(s). Overall average of DC voltage is $1.2 \times 10^{-6} \mathrm{VDC}$ and overall maximum is $11.78 \times 10^{-6}$ VDC. As average TGMR resistance is about $300 \mathrm{Ohms}$, so maximum calculated power on TGMR is $4.8 \times 10^{-13}$ Watts or 0.48 pico Joule/second. Next, this should be compared with threshold of TGMR failure which is typically susceptible to the energy in nano Joule range.

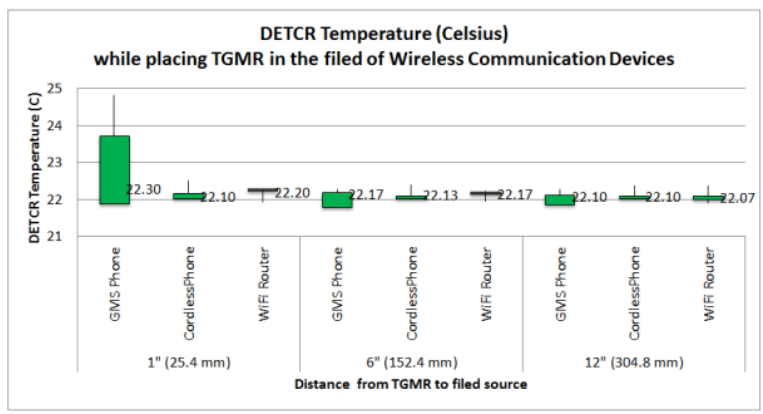

Figure6.1. Temperature of TGMR head while placing in the field of wireless communication device

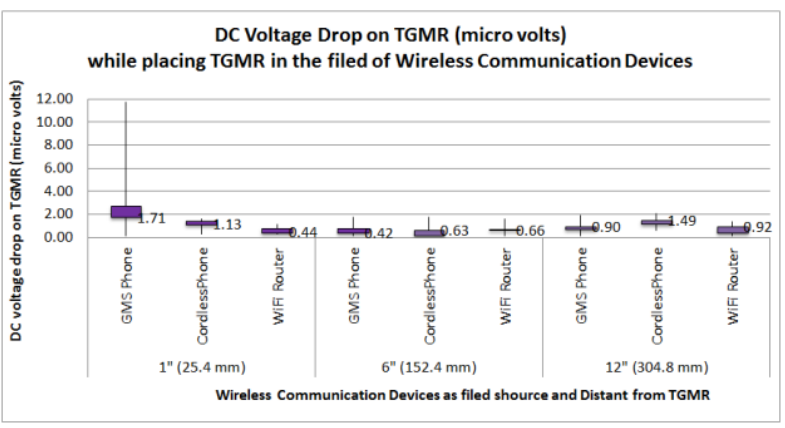

Figure6.2. DC voltage of TGMR head while placing in the field of wireless communication device

\subsection{Electrical Performance of TGMR pre and post actions simulating HGA assembly process}

Four types of wireless communication devices: GSM Cellular Phone, Cordless Phone, WiFi Router and Tablet Computer have been chosen to be field sources in this experiment because they are mostly used nowadays. HGAs are placed in the field generated from each device at D (cm.) distance far away as set up shown in Figure5.

Four actions of HGA process have been simulated during the HGAs are in-field as shown in Figure 7. There are; (I) No contact to HGA at all (II) Grounded-Metal 10 times contact to open traces of TGMR (III) GroundedNon-Metal 10 times contact to open traces of TGMR (IV)10 times pick-and-place on base plate with NonMetal End-Effector

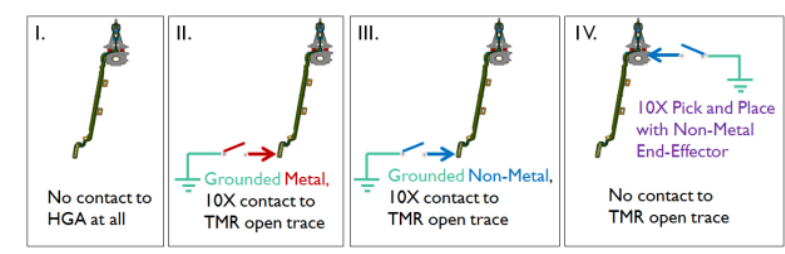

Figure7. Four actions represent HGA process

Electrical performance of HGAs has been tested before and after the actions with Quasi-static Tester (QST) to see how the experiment impacts to electrical performance of HGAs. Both catastrophic failure parameters (TGMR resistance, Amplitude, Slope) and instability parameters (Asymmetry, Barkhausen Jump, Hysteresis Loop, and Spectral Maximum Amplitude Noise/ S.M.A.N.) were observed and compared with total sample size of 180 HGAs. QST TGMR resistance measurement is static test and quite direct to see obvious physical change of TGMR sensor such as insulator or oxide layer breakdown. The other following parameters are measured and calculated from the Transfer Curve as shown in Figure8.

Amplitude is the key performance of reader sensor. The test measures peak amplitude in micro-volt at defined point of magnetic field. Amplitude is directly related to the change of TGMR resistance as shown in (3). While $I$ is the bias current, $V$ is the voltage drop on the 
sensor, $\Delta R / R_{0}$ is the intrinsic TGMR ratio, $\varepsilon$ is the efficiency or amount of free layer rotation. External energy such as Thermal, EOS/ESD can cause Pinned Layer reversal in TGMR and Slope test can reveal the magnitude and location of reversal over the sweep test range. Asymmetry test basically shows the symmetry of the transfer curve which can indicate performance of magnetic layers in TGMR. Equation (4) shows calculation of asymmetry from absolute value of the maximum and minimum amplitude. Hysteresis test is measurement area between forward amplitude (Fi) and reverse amplitude (Ri) of the amplitude. Equation (5) shows calculation of hysteresis, in the unit of microvolt/Oe, from amplitude and magnetic field increment (Inc). Barkhausen-jump (micro-volt) is maximum amplitude jumping between two adjacent measurement points. Percent of Barkhuasen jump $(\% B J)$ can be calculated with peak-to-peak amplitude ( $A m p \cdot p k-p k)$ as shown in (6). Spectral Maximum Amplitude Noise (S.M.A.N.) test measures noise density by sweeping through a range of DC magnetic fields. This parameter is invented to provide capability of high frequency instability identification. [11] [12] Example of S.M.A.N. test is shown in Figure9.

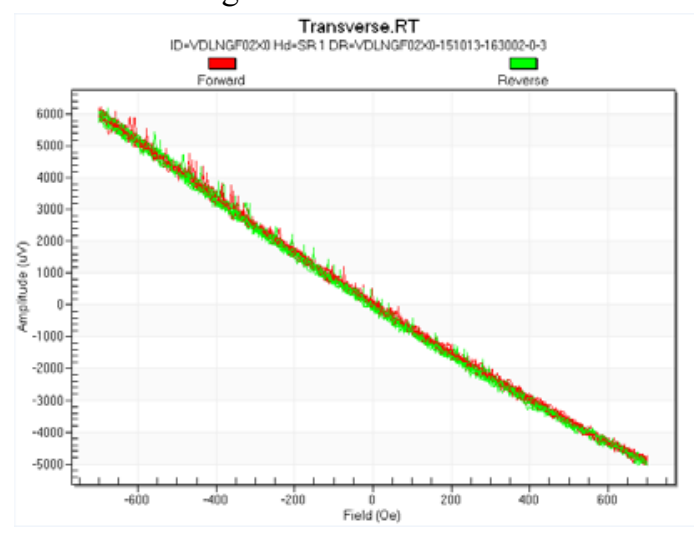

Figure8. Example of Amplitude Test by QST

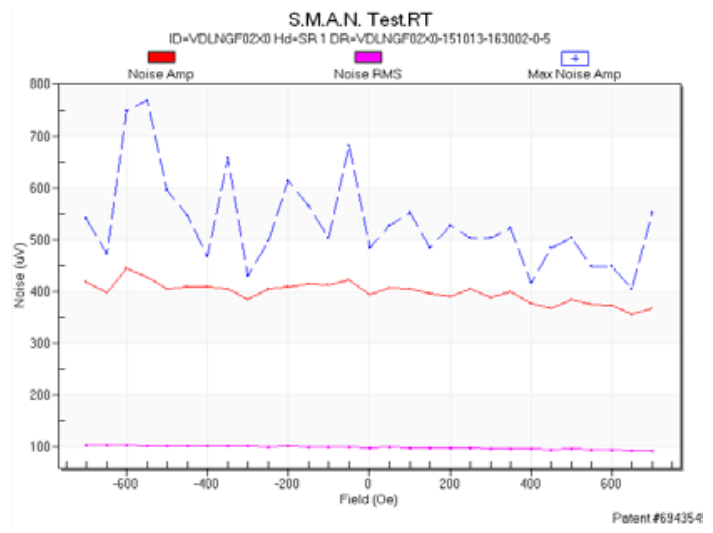

Figure9. Example of SMAN test by QST

Amplitude $=I \times \Delta R \times \varepsilon=I \times R_{0} \times \frac{\Delta R}{R_{0}} \times \varepsilon=V \times \frac{\Delta R}{R_{0}} \times \varepsilon$

$$
\begin{gathered}
\text { Asymmetry } \%)=\frac{|M A X \cdot a m p|-|M I N \cdot a m p|}{|M A X \cdot a m p|+|M I N \cdot a m p|} \times 100 \\
\text { Hysteresis }(\mu V / O e)=I n c \times \sum|F i-R i| \\
\% B J=[B J(\mu V) / A m p \cdot P k-P k \\
\% B 100
\end{gathered}
$$

The result from QST shows that, both catastrophic failure and instability failure parameters related to TGMR electrical performance (TGMR resistance, Amplitude, Slope, Asymmetry, Barkhausen Jump, Hysteresis-Loop, and Spectral Maximum Amplitude Noise) are not significantly changed. It is illustrated by correlation plot between pre and post actions simulating HGA assembly processes, in Figure10.1 to Figure10.7. This could confirm with the result of measuring Thermal change and DC voltage of TGMR head while placing in the field of wireless communication device in the section 3.2.

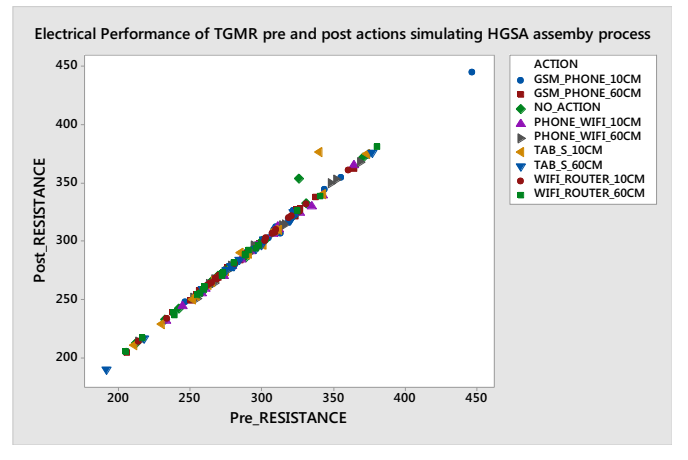

Figure10.1 Correlation of "TGMR Resistance"

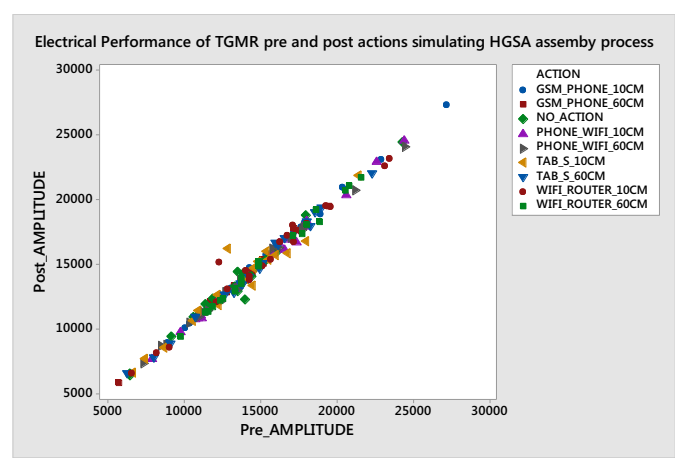

Figure10.2 Correlation of "TGMR Amplitude"

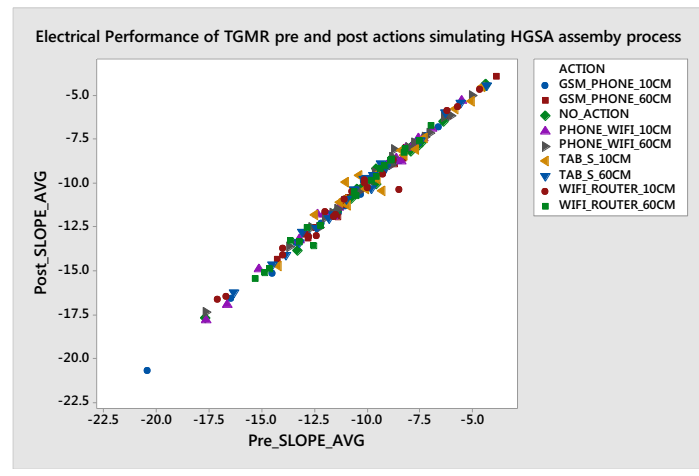

Figure10.3 Correlation of "TGMR Slope" 


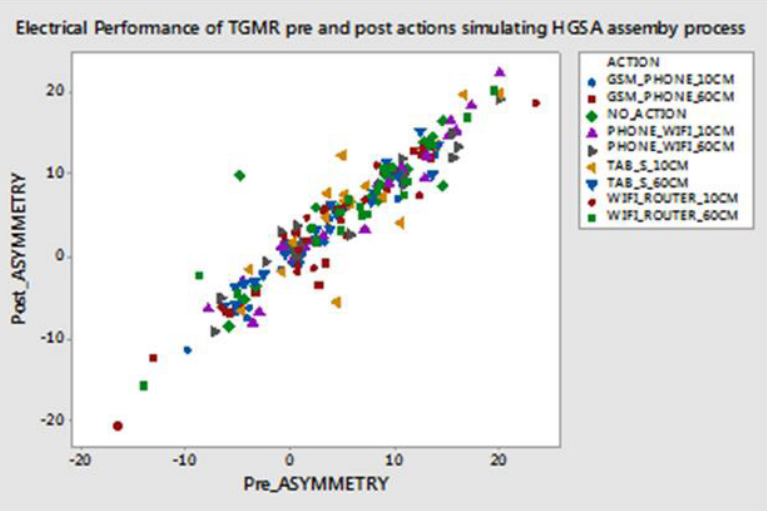

Figure10.4 Correlation of "Asymmetry"

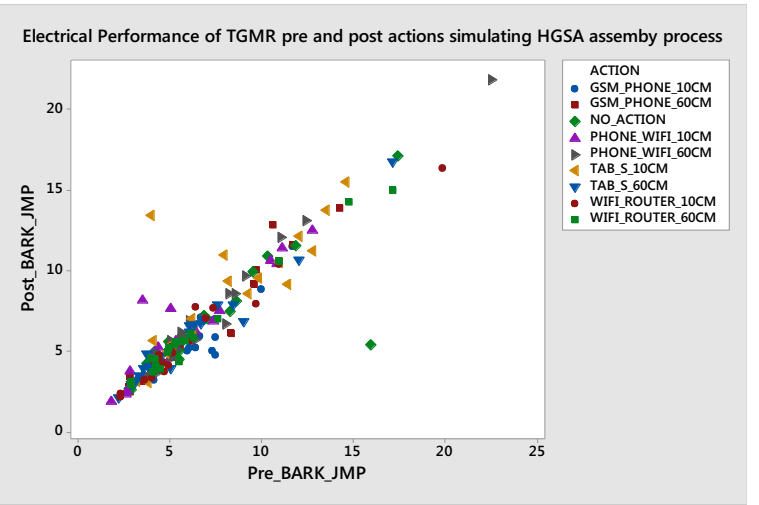

Figure10.5 Correlation of "Barkhausen Jump"

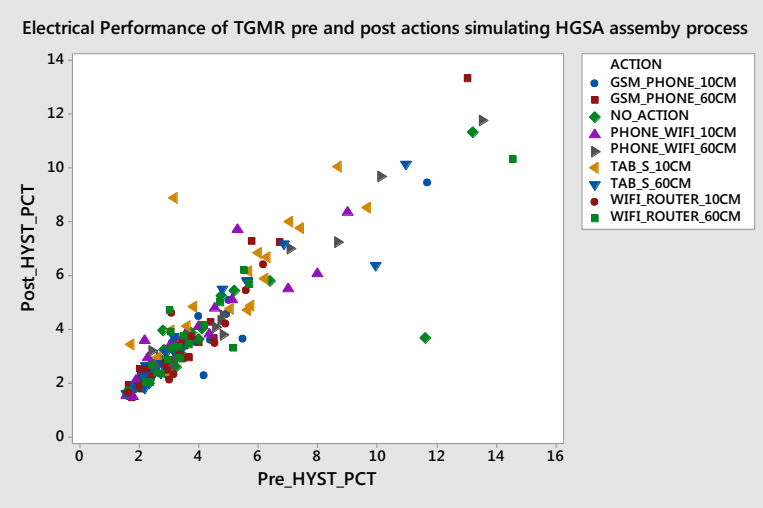

Figure.10.6 Correlation of "Hysteresis Loop"

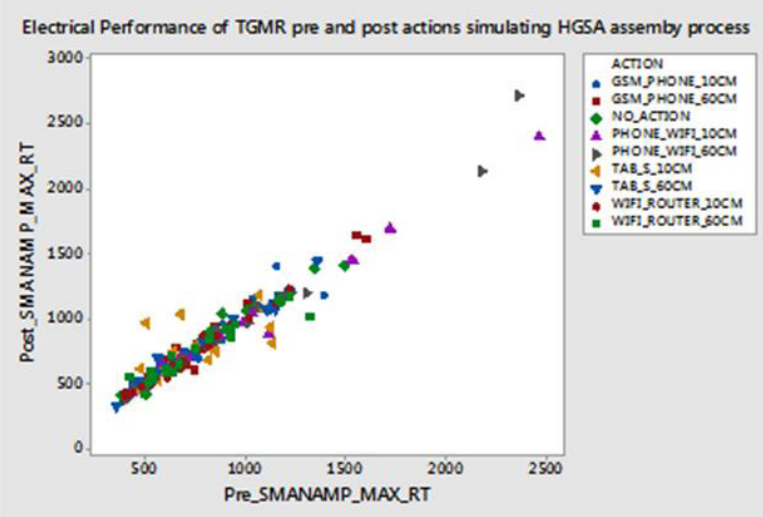

Figure10.7 Correlation of "S.M.A.N."

\section{Conclusions}

In this study, Electromagnetic Interference of 1-16 V/m had been observed from GSM cell phone, Cordless Phone, WiFi Router and Tablet Computer. However, when placing TGMR head in the electromagnetic fields issued from those wireless communication devices, the thermal change of TGMR head and the DC voltage of TGMR are not significant to create instability nor catastrophic failure to TGMR heads.

It had been proved with key QST parameters on 180 HGA samples for pre and post actions simulating HGA assembly process in the field of those wireless communication devices. There is no significant change in TGMR resistance, Amplitude, Slope, Asymmetry, Barkhausen Jump, Hysteresis Loop, and Spectral Maximum Amplitude Noise (S.M.A.N.) in all experiment conditions.

Wireless communication devices may be considered to enhance operation efficiency or operation cost saving. Nevertheless, it should be studied to make sure the effect on energy sensitive devices as well as automated production equipment and testers to be in acceptable level.

\section{Acknowledgements}

This work was supported by; [i] Seagate Technology, PLC, [ii] Seagate Technology, Thailand, Ltd, [iii] National Electronics and Computer Technology Center (NECTEC) Thailand, [iv] College of Data Storage Innovation, King Mongkut's Institute of Technology Ladkrabang, [v] Faculty of Engineering, King Mongkut's Institute of Technology Ladkrabang, Thailand.

\section{References}

1. Wallash, D. Smith, EOS/ESD Symposium 98, 4B.6.1 (1998).

2. C.F. Lam, D. Martinez, C. Chang, EOS/ESD Symposium 99, 4B.6.2 (1999).

3. V. Kraz, A. Wallash, EOS/ESD Symposium 00, 2C.10.1 (2000).

4. S. Natori, T. Wada, Y. Itou, H. Sakata, K. Kataoka, H. Tanabe, T. Ohtsu, EOS/ESD Symposium 06, 4B.3.1 (2006).

5. S. Kaengrang, A. Kaewrawang, DSTCON 4th, pp. 61 (2011).

6. S. Puapairoj, C. Sa-ngiamsak, DSTCON 4th, pp. 149 (2011).

7. V. Kraz, P. Tachamaneekorn, D. Napombejara, EOS/ESD Symposium 05, 4A.4.1 (2005).

8. T. Zhao, K. Zhang, H. C. Wang, Y.H. Chen, M. Li, US Patents, US20070111332 A1 (2007).

9. M.A. Dufresne, B.W. Karr, G.J. Kunkel, Z. Wei, US Patents, US20120120982 A1 (2012).

10. D. Liu, K. Haapala, S.E. Ryun, L. Zhou, US Patents, US8638349 B1(2014).

11. H.Patland, W.A. Ogle, US6943545 B2 (2005)

12. H.Patland, W.A. Ogle, EOS/ESD Symposium 02, pp. 130 (2002). 